\title{
Perfil clínico epidemiológico de los pacientes con cáncer tratados en una institución de tercer nivel. Manizales, Colombia, 1995-2004
}

\author{
Epidemic clinical profile of cancer patients treated in a third-level \\ clinical institution in Manizales, Colombia, 1995-2004
}

\author{
Carlos Raúl Villegas, MD ${ }^{1}$, José Arnoby Chacón, $\mathrm{MSc}^{2}$, \\ Juan Paulo Cardona, MD ${ }^{3}$, Luz Ángela Correa, Enf ${ }^{4}$
}

\section{Resumen}

Antecedentes: Los diagnósticos de cáncer son cada vez mayores y ello está asociado con el aumento de la expectativa de vida, la exposición a carcinógenos, la cobertura en salud, el tamizaje y la búsqueda activa de casos. En Estados Unidos se diagnostican 1’000,000 de casos nuevos y más de 500,000 muertes por año. Los costos de la atención y la incapacidad laboral son muy altos. En Colombia, el patrón de ocurrencia de esta enfermedad se constituye en un problema de salud pública. En Manizales, en el año 2004, se diagnosticaron 653 casos nuevos.

Objetivo: Describir la población con diagnóstico de cáncer atendida en el Instituto Oncológico (ION SA) de Manizales, Colombia, desde su fundación en el año 1995, hasta diciembre de 2004.

Métodos: Se incluyeron 5,000 pacientes tratados durante 1995 a 2004. Las variables demográficas y clínicas se analizaron para establecer las frecuencias de presentación y los principales aspectos del manejo y los resultados del tratamiento.

Resultados: Edad promedio 57 años \pm 17.3 ; sexo femenino (65.6\%); procedencia urbana (90.2\%). La principal categoría ocupacional fue «Oficios varios» (61\%) seguida de «Técnico» (12.1\%). Los diagnósticos con mayor frecuencia fueron cáncer de mama (18.7\%), cáncer de cérvix (13.1\%), linfomas (7.7\%), cáncer colorectal (6.4\%) y cáncer gástrico (4.7\%). La mayoría de los pacientes (69.1\%) consultó después de dos meses de presentar síntomas. Las etapas clínicas III y IV fueron las más frecuentes en el momento de la consulta. Según la escala de Karnofsky, la capacidad funcional era buena al inicio del tratamiento. El manejo general se hizo con cirugía, quimioterapia y radioterapia. La toxicidad medicamentosa se presentó en 11.3\% (567/5,000). La supervivencia a 5 años para hombres y mujeres fue de $61.2 \%$ y $72.3 \%$, respectivamente $(\mathrm{p}<0.05)$.

Conclusión: El perfil clínico y epidemiológico de los pacientes atendidos en el ION SA, mantiene las tendencias mundialmente determinadas por otros estudios institucionales. Se resalta la posibilidad de hacer análisis secundarios, porque el registro institucional puede conducir a la mejor comprensión del fenómeno local y de otras áreas de atención oncológica.

Palabras clave: Cáncer; Sobrevida; Tratamiento.

Colomb Med. 2012; 43: 11-18

\footnotetext{
Oncólogos del Occidente SA, Manizales, Colombia. e-mail: caravim@hotmail.com

2 Profesor Titular, Facultad de Ciencias para la Salud, Departamento Clínico, Universidad de Caldas, Manizales, Colombia. e-mail:jose.chacon@ucaldas.edu.co

ProfesorAuxiliar, Facultad de Ciencias para la Salud, Departamento Clínico, Universidad de Caldas, Manizales, Colombia. e-mail: jpcardona2@yahoo.com

4 Enfermera, Facultad de Ciencias para la Salud, Departamento Clínico, Universidad de Caldas, Manizales, Colombia. e-mail: luz.correa@ucaldas.edu.co

Recibido para publicación octubre 28, 2010 Aceptado para publicación agosto 10, 2011
} 


\section{Summary}

Background: Cancer is being increasingly diagnosed throughout the world, possibly related to increased life expectancy, greater exposure to known carcinogens, increased healthcare coverage, application of screening programs, and active search for cases. In the United States, 1-million new cases are diagnosed with more than 500,000 deaths per year. The costs of attention and work lost are very high. In Colombia, this pathology has behaved in similar manner, constituting a general public health problem. In 2004, in Manizales 653 new cases were diagnosed.

Objective: To describe the characteristics of the population with cancer diagnosis treated in the Instituto Oncológico ION SA of Manizales, Caldas, Colombia, since its foundation on March/1995 to December/2004.

Materials and methods: Some 5000 patients were treated in the Institution were included from 1995 to 2004. The demographic and clinical variables were analyzed to establish the frequencies of presentation and to know the main aspects of the study given in the Institution and the most important results about the treatment with different therapeutic modalities.

Results: Median age of 57 years \pm 17.3 , female $(65.6 \%)$, urban origin $(90.2 \%)$. The main occupational category was «Various occupations» (61\%) followed by «Technical» $(12.1 \%)$. The most common diagnoses were breast cancer $18.7 \%$, cervical cancer $13.1 \%$, lymphomas $7.7 \%$, colorectal cancer $6.4 \%$, and gastric cancer $4.7 \%$. Most patients $(69.1 \%)$ consulted after two months of their first symptoms. The III and IV clinical stages were the most frequent at diagnosis. According to the Karnofsky scale, the functional capacity was good at the beginning of treatment. Therapeutic modalities were surgery, chemotherapy, and radiotherapy. The toxicity of the treatments revealed a total frequency of $11.3 \%$ (567/ $5,000)$. The overall survival at 5 years for men and women was $61.2 \%$ and $72.3 \%$, respectively $(\mathrm{p}<0.05)$.

Conclusions: The clinical and epidemic profile of the patients attended in the ION SA in Manizales, Colombia, does not differ in its general aspect from other cancer studies, other analytic studies must be conducted to determine associations among them to help to best understand the Oncologic phenomenon of our Institution.

\section{Keywords: Cancer; Survival; Treatment. Colomb Med. 2012; 43: 11-18}

El cáncer, como problema de salud pública, es creciente en el mundo. El aumento en la expectativa de vida de las poblaciones, la mayor exposición a carcinógenos reconocidos, la ampliación de la cobertura de los programas de salud, el continuo mejoramiento de las técnicas de tamizaje y la búsqueda activa de casos, son las razones por las cuales anualmente se informa un aumento en el número global de casos diagnosticados con cáncer. Según datos del Instituto Nacional de Salud de Estados Unidos, en este país se diagnostican 1'000.000 de casos nuevos cada año, con más de 500,000 muertes en el mismo período. Se estima que 35\% de la población sufrirá un tumor maligno, es decir 76 millones de personas. Anualmente se invierten 22,000 millones de dólares en costos médicos directos y se dedican 50,000 millones de dólares en incapacidades laborales ${ }^{1}$.

El Instituto Nacional de Cancerología de Colombia reportó para el quinquenio 2002-2006 una incidencia de 3.2316 casos en hombres y 3.8571 casos en mujeres en Colombia. La mortalidad para este quinquenio fue de 72,529 en hombres y 75,536 en mujeres, lo que representa una tasa de 84.4 por 100,000 en hombres y 72.2 por 100,000 en mujeres. Según el Registro Poblacional para la Vigilancia Epidemiológica del Cáncer en el departamento de Caldas (área de influencia del Instituto Oncológico ION), publicado en diciembre del año 2009, resultados del quinquenio 2002-2006 se diagnosticaron 1,542 casos nuevos en Manizales $(60.9 \%$ ) de 2,530 casos nuevos en el departamento de Caldas durante el período 20022006, de los que 57.6\% corresponde a la población femenina.

Con el fin de describir las características de la población atendida con diagnóstico de cáncer y ubicarla en el contexto de la problemática mundial y nacional, se revisaron los registros médicos de los pacientes del Instituto Oncológico ION de Manizales, Colombia, desde su fundación, en el año 1995, hasta diciembre de 2004. 


\section{Materiales y métodos}

Mediante un estudio de tipo descriptivo se analizaron 5,000 casos de pacientes con diagnóstico de cáncer de quienes se obtuvo información mediante un registro institucional desde 1995. Las variables se agruparon según características demográficas y clínicas. En el primer grupo se tuvieron en cuenta la procedencia, el sexo, la edad y la ocupación. Dentro de las variables clínicas se consideraron aquellas que reflejan las condiciones de llegada a la institución, las medidas terapéuticas realizadas y el estado general al término del manejo institucional.

Se utilizó una base de datos que se ha diligenciado mediante el programa EpiInfo 6.0. Las variables cualitativas se presentan a través de proporciones y las cuantitativas con promedios y desviaciones estándar. El proyecto fue avalado por el Comité de Ética e Investigaciones del Instituto Oncológico y permitió el uso de su razón social en el texto.

\section{Resultados}

Del total de pacientes $65.6 \%$ son mujeres. El promedio de edad fue 54.2 años (DE 17.3), distribuidos por grupos con fines de comparación (Cuadro 1). La distribución etárea en tres grandes grupos muestra una diferencia significativa en la frecuencia del cáncer $(\mathrm{p}<0.001)$. También se dividió el grupo en $\leq 35$ años y mayores, para el análisis de mortalidad proporcio-

\section{Cuadro 1}

Características demográficas de los pacientes tratados en ION SA 1995-2004

\begin{tabular}{lccc}
\hline \multirow{2}{*}{ Característica } & \multicolumn{3}{c}{ Número y porcentaje (\%) } \\
\cline { 2 - 4 } & Total $(\mathbf{n = 5 0 0 0 )}$ & Mujeres (n=3280) & Hombres (n=1720) \\
\hline Edad (años) & & & \\
$<45$ & $1419(28.4)$ & $913(27.8)$ & $506(29.4)$ \\
$45-60$ & $1593(31.9)$ & $1198(36.5)$ & $395(23.0)$ \\
$>60$ & $1988(39.8)$ & $1169(35.6)$ & $819(47.6)$ \\
Edad especial (años) & & & $334(19.4)$ \\
$\leq 35$ & $662(13.2)$ & $328(10.0)$ & $1386(80.6)$ \\
$>35$ & $4338(86.8)$ & $2952(90.0)$ & $212(12.3)$ \\
Zona residencia & & & $1508(87.7)$ \\
Rural & $492(9.8)$ & $280(8.5)$ & $103(6.0)$ \\
Urbana & $4508(90.2)$ & $3000(91.5)$ & $319(18.5)$ \\
Grupo ocupación & & & $40(2.3)$ \\
Profesional & $281(5.6)$ & $178(5.4)$ & $547(31.8)$ \\
Técnico & $603(12.1)$ & $284(8.7)$ & $431(25.1)$ \\
Auxiliar & $100(2.0)$ & $60(1.8)$ & $35(2.0)$ \\
Oficios varios & $3048(61.0)$ & $2501(76.3)$ & $315(6.7)$ \\
Pensionado & $575(11.5)$ & $144(4.4)$ & $6(0.3)$ \\
Independiente & $41(0.8)$ & $80(2.4)$ & $7(0.2)$ \\
Estudiante & $195(3.9)$ & $20(0.6)$ & \\
Desempleado & $98(2.0)$ & & \\
Menor de edad & $59(1.2)$ & & \\
\hline
\end{tabular}


nal. El $90.2 \%$ de los pacientes procede de zona urbana, lo que concuerda con la distribución espacial de la población del área de influencia del Instituto ION. La ocupación se agrupó en «categorías ocupacionales» teniendo en cuenta el nivel de instrucción alcanzado y la dedicación en el momento de la atención inicial.

La descripción de las variables clínicas se hizo a partir de la primera consulta en la institución desde el inicio de sus labores asistenciales (Cuadro 2). Los grupos diagnósticos siguen la clasificación general de diferentes informes de orden nacional e internacio$\mathrm{nal}^{2}$. Las mayores frecuencias de diagnóstico en las mujeres fueron: mama, cérvix, colorrectal y linfomas, y en los hombres: linfomas, próstata, estómago y cáncer colorrectal. Se presentaron diferencias significativas entre sexos para algunos tipos de cáncer (Cuadro 2) ${ }^{1}$.

La duración de los síntomas antes del diagnóstico se agrupó en 5 categorías y en ellas se aprecia que 69.1\% de los pacientes presentaba síntomas con más de dos meses de duración, lo cual puede reflejar dificultades de accesibilidad al sistema de salud y, más específicamente, a la consulta especializada.

En cuanto al género se encuentra que un mayor porcentaje de las mujeres consultó en los primeros dos meses de sintomatología $(33.1 \%$ vs. $26.7 \%)$ $(\mathrm{p}<0.001)$. Llama la atención que en el grupo de duración de síntomas entre dos y seis meses, se invierte el patrón de ocurrencia con mayor porcentaje de hombres $(28.9 \%$ vs. $25.0 \%)(\mathrm{p}<0.01)^{3}$. Etapas clínicas localizadas I y II en $26.6 \%$ al ingreso de los pacientes al Instituto para su tratamiento; 86.7\% presentó una clasificación en la escala de Karnofsky al inicio del tratamiento de $80 \%$ o mayor.

El tratamiento se describe en forma general con radioterapia, quimioterapia y formas mixtas y no se tienen en cuenta las especificidades de cada tumor. La tolerancia al tratamiento se clasificó mediante una apreciación subjetiva del paciente en tres categorías sin encontrar diferencias importantes entre ellas. Los resultados de la categoría «buena» son mejores en las mujeres $(65.9 \%)$ que en los hombres $(34.1 \%)$
( $\mathrm{p}<0.001)$, lo que puede estar en relación con la percepción general de que los hombres toleran menos los procedimientos médicos. Al finalizar el tratamiento se aplicó de nuevo la escala de Karnofsky y se encontró que el estado general de los pacientes no varió con respecto al inicio de la terapia.

El 11.34\% de los pacientes $(567 / 5,000)$ presentó alguna toxicidad, predominó el compromiso hematológico, de mucosas y de sistema digestivo. Se presentó diferencia significativa entre el género sólo en las complicaciones de tipo digestivo. En $25.6 \%$ de los casos se presentó complicaciones en varios sistemas.

El seguimiento médico de los pacientes se ha hecho en el Instituto y con el protocolo de vigilancia oncológica, desde su fundación, en mayo de 1995, hasta la fecha del presente estudio, con un promedio de 16.8 meses (rango 1 a 110 meses).

\section{Discusión}

Se analiza la experiencia de 10 años de trabajo de un registro institucional de pacientes que fueron atendidos en el Instituto Oncológico ION SA de la ciudad de Manizales con diagnóstico comprobado de cáncer. La frecuencia de casos aumenta paulatinamente desde 1995 como corresponde a una institución naciente y a unas condiciones de contratación de servicios con entidades aseguradoras de salud.

La presentación por sexo y edad muestra que en el grupo de menores de 45 años no hay diferencias significativas, pero, a partir de esa edad, son representativas las diferencias a expensas de una mayor frecuencia en hombres mayores de 60 años; situación distinta se presenta en informes mundiales ${ }^{4,5}$ y nacionales ${ }^{1,3}$ donde se obtiene una relación mujer a hombre de 1.2 a 1. En este trabajo se encuentra una relación mujer a hombre de 1:0.7.

El análisis por edad constituye una demostración del patrón general de ocurrencia del cáncer en el que, a mayor número de años hay mayores probabilidades de presentación, porque es una enfermedad crónica y de larga evolución. La presentación en menores de 


\section{Cuadro 2}

Características clínicas de los pacientes tratados en ION SA 1995-2004

\begin{tabular}{|c|c|c|c|}
\hline \multirow[t]{2}{*}{ Característica } & \multicolumn{3}{|c|}{ Número y porcentaje (\%) } \\
\hline & Total $(n=5000)$ & Mujeres (n=3280) & Hombres $(n=1720)$ \\
\hline \multicolumn{4}{|l|}{ Año diagnóstico } \\
\hline 1995 & $122(2.4)$ & $86(2.6)$ & $36(2.1)$ \\
\hline 1996 & $278(5.6)$ & $199(6.1)$ & $79(4.6)$ \\
\hline 1997 & $434(8.7)$ & $281(8.6)$ & $153(8.9)$ \\
\hline 1998 & $430(8.6)$ & $250(7.6)$ & $180(10.5)$ \\
\hline 1999 & $412(8.2)$ & $253(7.7)$ & $159(9.2)$ \\
\hline 2000 & $487(9.7)$ & $331(10.1)$ & $156(9.1)$ \\
\hline 2001 & $514(10.3)$ & $331(10.1)$ & $183(10.6)$ \\
\hline 2002 & 1193 (23.9) & 835 (25.5) & $358(20.8)$ \\
\hline 2003 & $502(10.0)$ & $319(9.7)$ & $183(10.6)$ \\
\hline 2004 & $617(12.3)$ & $392(11.9)$ & $233(13.2)$ \\
\hline \multicolumn{4}{|l|}{ Diagnóstico } \\
\hline Ca cérvix & $655(13.1)$ & $655(20.0)$ & 0 \\
\hline Ca colorrectal & $319(6.4)$ & $180(5.5)$ & $139(8.1)$ \\
\hline Ca esófago & $63(1.3)$ & $35(1.1)$ & $28(1.6)$ \\
\hline Ca estómago & $237(4.7)$ & $77(2.3)$ & $160(9.3)$ \\
\hline Ca hígado & $52(1.0)$ & $41(1.3)$ & $11(0.6)$ \\
\hline Ca mama & 933 (18.7) & $924(28.2)$ & $9(0.5)$ \\
\hline Ca oral & $17(0.3)$ & $4(0.1)$ & $13(0.8)$ \\
\hline Ca páncreas & $25(0.5)$ & $14(0.4)$ & $11(0.6)$ \\
\hline Ca próstata & $183(3.7)$ & 0 & $183(10.6)$ \\
\hline Ca traq/bron/pul & $218(4.4)$ & $78(2.4)$ & $140(8.1)$ \\
\hline Ca útero & $77(1.5)$ & $77(2.3)$ & 0 \\
\hline Ca vejiga & $35(0.7)$ & $6(0.2)$ & $29(1.7)$ \\
\hline Leucemia & $100(2.0)$ & $45(1.4)$ & $55(3.2)$ \\
\hline Linfomas & $386(7.7)$ & $178(5.4)$ & $208(12.1)$ \\
\hline Otros malignos & $1700(34.0)$ & $966(29.5)$ & $734(42.7)$ \\
\hline Duración síntomas & & & $459(26.7)$ \\
\hline$<2$ meses & $1544(30.9)$ & $1085(33.1)$ & \\
\hline 2 a 6 meses & $1316(26.3)$ & $819(25.0)$ & 497 (28.9) \\
\hline 7 a 12 meses & $1104(22.1)$ & $705(21.5)$ & $399(23.2)$ \\
\hline 13 a 24 meses & $560(11.2)$ & $360(11.0)$ & $200(11.6)$ \\
\hline$>24$ meses & $476(9.5)$ & $311(9.5)$ & $165(9.6)$ \\
\hline \multicolumn{4}{|l|}{ Etapa clínica } \\
\hline 1 & $589(11.8)$ & $360(11.0)$ & $229(13.3)$ \\
\hline II & 739 (14.8) & 580 (17.7) & $159(9.2)$ \\
\hline III & $1042(20.8)$ & $763(23.2)$ & $280(16.3)$ \\
\hline IV & $644(12.8)$ & $326(9.9)$ & 318 (18.5) \\
\hline No clasificado & $1291(25.8)$ & 777 (23.7) & $514(29.9)$ \\
\hline Desconocido & $695(13.9)$ & $475(14.5)$ & $220(12.8)$ \\
\hline \multicolumn{4}{|l|}{ Karnofsky inicial (\%) } \\
\hline$<40$ & $25(0.5)$ & $16(0.5)$ & $9(0.5)$ \\
\hline 50 a 70 & $636(12.7)$ & $321(9.8)$ & $315(18.3)$ \\
\hline$>80$ & $4331(86.7)$ & $2936(89.6)$ & $1395(81.1)$ \\
\hline Desconocido & $5(0.1)$ & $4(0.12)$ & $1(0.6)$ \\
\hline \multicolumn{4}{|l|}{ Tratamiento Ion SA } \\
\hline Cirugía & $1(0.0)$ & $1(0.0)$ & 0 \\
\hline Radioterapia & $1171(23.4)$ & $622(19.0)$ & 549 (31.9) \\
\hline Quimioterapia & $1029(20.6)$ & $696(21.2)$ & $333(19.4)$ \\
\hline Mixto & 1107 (22.1) & $819(25.0)$ & $288(16.7)$ \\
\hline Paliativo & $1692(33.8)$ & $1142(34.8)$ & $556(32.0)$ \\
\hline
\end{tabular}




\section{Cuadro 2}

Características clínicas de los pacientes tratados en ION SA 1995-2004 (continuación)

\begin{tabular}{lccc}
\hline Característica & \multicolumn{3}{c}{ Número y porcentaje (\%) } \\
\cline { 2 - 4 } & Total $(\mathbf{n = 5 0 0 0 )}$ & Mujeres $(\mathbf{n = 3 2 8 0})$ & Hombres $(\mathbf{n = 1 7 2 0})$ \\
\hline Radioterapia & $(\mathrm{n}=3081)$ & $(\mathrm{n}=2082)$ & $(\mathrm{n}=999)$ \\
$\quad$ Teleterapia & 2644 & $1648(79.1)$ & $996(99.7)$ \\
Braquiterapia & 7 & 7 & 0 \\
$\quad$ Mixto & 430 & $427(20.5)$ & $3(0.03)$ \\
Karnofsky final (\%) & & & \\
<40 & $50(1.0)$ & $34(1.0)$ & $16(0.9)$ \\
50 a 70 & $556(11.2)$ & $292(8.9)$ & $264(15.4)$ \\
$>80$ & $4249(85.3)$ & $2861(87.5)$ & $1388(81.0)$ \\
Desconocido & $129(2.6)$ & $84(2.6)$ & $45(2.6)$ \\
Tolerancia tratamiento & & & \\
Buena & $4624(92.5)$ & $3047(92.9)$ & $1577(91.7)$ \\
Regular & $313(6.3)$ & $190(5.8)$ & $123(7.2)$ \\
Mala & $43(0.9)$ & $30(0.9)$ & $13(0.8)$ \\
Desconocida & $20(0.4)$ & $13(0.4)$ & $7(0.4)$ \\
Toxicidad & $\mathrm{n}=567$ & $\mathrm{n}=362$ & $\mathrm{n}=205$ \\
Hematológica & $135(23.8)$ & $96(26.5)$ & $39(19.0)$ \\
Otra & $19(3.4)$ & $12(3.3)$ & $7(3.4)$ \\
Mixta & $145(25.6)$ & $93(25.7)$ & $52(25.4)$ \\
Digestiva & $68(12.0)$ & $14(13.3)$ & $20(9.8)$ \\
Neurológica & $10(1.8)$ & $8(2.2)$ & $2(1.0)$ \\
Neumológica & $13(2.3)$ & $5(1.4)$ & $8(3.9)$ \\
Cutánea & $43(7.6)$ & $22(6.1)$ & $21(10.2)$ \\
En mucosas & $104(18.3)$ & $57(15.7)$ & $47(22.9)$ \\
Ósea & $3(0.5)$ & $2(0.6)$ & $1(0.5)$ \\
\hline
\end{tabular}

45 años para todos los tumores en el Instituto, muestra una mayor frecuencia de presentación en etapas más tempranas que en lo reportado en Colombia $^{1}$.

Los dos tumores más frecuentes fueron el de mama y el de cérvix, de muy mal pronóstico por su carácter agresivo a pesar de los esfuerzos terapéuticos con mortalidad mayor a $60 \%{ }^{1,3,4}$.

Los cinco primeros diagnósticos, como en otros análisis locales y regionales presentan una distribución por sexo similar a países en desarrollo $0^{5,7}$. La presentación del cáncer de mama en el sexo masculino es similar a otras series de la literatura oncológica general, semejante a lo reportado en el Registro Poblacional para Caldas ${ }^{3,6}$. Los cinco primeros diagnósticos, como en otros análisis locales y regionales, presentan una distribución por sexo similar a países en desarrollo ${ }^{5,7}$.

Si se hace una comparación en dos series institucionales latinoamericanas con características sociodemográficas similares, como es el caso del Instituto Nacional de Cancerología (INCAN), México y la del Instituto Oncológico ION, entre las mujeres se encuentran diferencias en la presentación de cáncer de cérvix, mama, linfoma, útero, distinta a la presentación en $\mathrm{ION}^{7}$.

Entre los hombres, en el INCAN, México, se presentan resultados diferentes en cáncer de testículo, cáncer de pulmón, linfoma, próstata y el cáncer de cavidad oral y se excluye el de glándula salival mayor, con respecto a la presentación en la serie en ION con el linfoma en el primer lugar, próstata, estómago, pulmón y cáncer colorrectal.

Llama la atención la presentación del cáncer primario de origen desconocido en la población mexicana frente a la población de ION en Manizales con una diferencia más evidente en el sexo masculino ${ }^{7}$.

El análisis por ocupación muestra una mayor 
participación del grupo de «oficios varios», explicable por el tipo de aseguramiento y la contratación con el Instituto de Seguros Sociales, que fue el organismo de seguridad social más grande en el país y que tradicionalmente ha afiliado a este grupo de trabajadores. Los grupos que siguen en frecuencia son los «técnicos» y los «pensionados», por lo que podría pensarse en una mayor exposición relacionada con sus actividades laborales ${ }^{8,9}$.

El mayor aporte lo hace el área urbana, lo que refleja la distribución de la población del área de influencia del instituto similar a otras series relacionadas en la literatura ${ }^{3,7}$.La etapa clínica refleja el estado de los pacientes a la hora de buscar atención médica. Los resultados del presente estudio son comparables con los de la población colombiana en general; los estadíos clínicos se presentaron en orden decreciente en avanzados, localizados, los definidos como «no clasificados», que representan aquellos pacientes que sufrieron algún tipo de manipulación diagnóstica o terapéutica previo al ingreso al Instituto y el grupo de «desconocido». Los datos de estas dos últimas categorías pueden ser una manifestación de la idiosincrasia y un reflejo de la situación médico administrativa de los países en desarrollo ${ }^{1}$.

Como una manifestación de condiciones sociales, económicas, laborales, de comunicación, transporte, referencia y contra referencia de pacientes para llegar a un centro especializado de atención, se encontró que los pacientes refieren síntomas, en su mayoría, con más de 6 meses, lo que coincide con lo referido por otros autores ${ }^{6}$. Todo lo anterior da cuenta de las condiciones del sistema actual de salud de Colombia, sin descartar una gran influencia cultural de la población.

La capacidad funcional de los pacientes al ingreso y la evaluación posterior al tratamiento muestra pocas diferencias, lo que sugiere que el resultado terapéutico inmediato no genera, por sí mismo, un deterioro en la condición del paciente establecido por la aplicación de la escala de Karnofsky al inicio y al finalizar el tratamiento. Esta condición también se refleja cuando se evalúa la tolerancia al tratamiento en la que los pacientes la califican en la categoría de «buena». Es preciso señalar que un tercio de los pacientes recibe tratamiento exclusivamente paliativo como consecuencia del estado inicial en el momento del ingreso al Instituto, lo cual concuerda con el hecho de que hay demora en la búsqueda de ayuda y tratamiento médico.

Durante el período analizado, en el Instituto se aplicó quimioterapia, radioterapia y tratamiento combinado de quimioterapia y radioterapia. Del total de pacientes tratados se encontraron con manifestaciones de toxicidad tales como mixtas, hematológicas (derivadas de la aplicación de quimioterapia, como anemia y freno medular de grado I-II-III), en mucosas (mucositis orales principalmente grado I). En la serie de éste estudio se encontraron complicaciones digestivas como diarrea, náuseas, emesis y mucositis del tipo proctitis y rectitis actínica diferente a lo referido en la literatura, relacionadas con los tratamientos de quimioterapia y radioterapia concomitante. La presencia de alteraciones cutáneas del tipo radiodermitis actínica grado I se relaciona con el uso de radioterapia $^{1,10}$.

El control de los pacientes se hizo en promedio durante 16.9 meses $(\mathrm{DE}=22.9)$ y el análisis de supervivencia presente en la Gráfica 1 mostró una mejor sobrevida en mujeres con respecto a los hombres.

Perfil. El perfil de los pacientes tratados en la institución de tercer nivel de salud de la ciudad de Manizales se determina como resultado de este estudio, así: Desde el punto de vista demográfico se trata de un paciente mayor de 60 años, procedente de la zona urbana de la ciudad de Manizales, de sexo femenino con ocupación «oficios varios». Las características clínicas del paciente en su primer contacto con esta institución, son: diagnóstico de cáncer de mama o de cuello uterino, con una duración de síntomas menor de seis meses, que se encuentra en etapa clínica III con Karnofsky mayor de 80\%, que recibe tratamiento mixto (radioterapia y quimioterapia) y que al final del tratamiento no presenta cambios en el índice de Karnofsky. Durante el tratamiento presenta muy buena tolerancia, con manifestaciones 


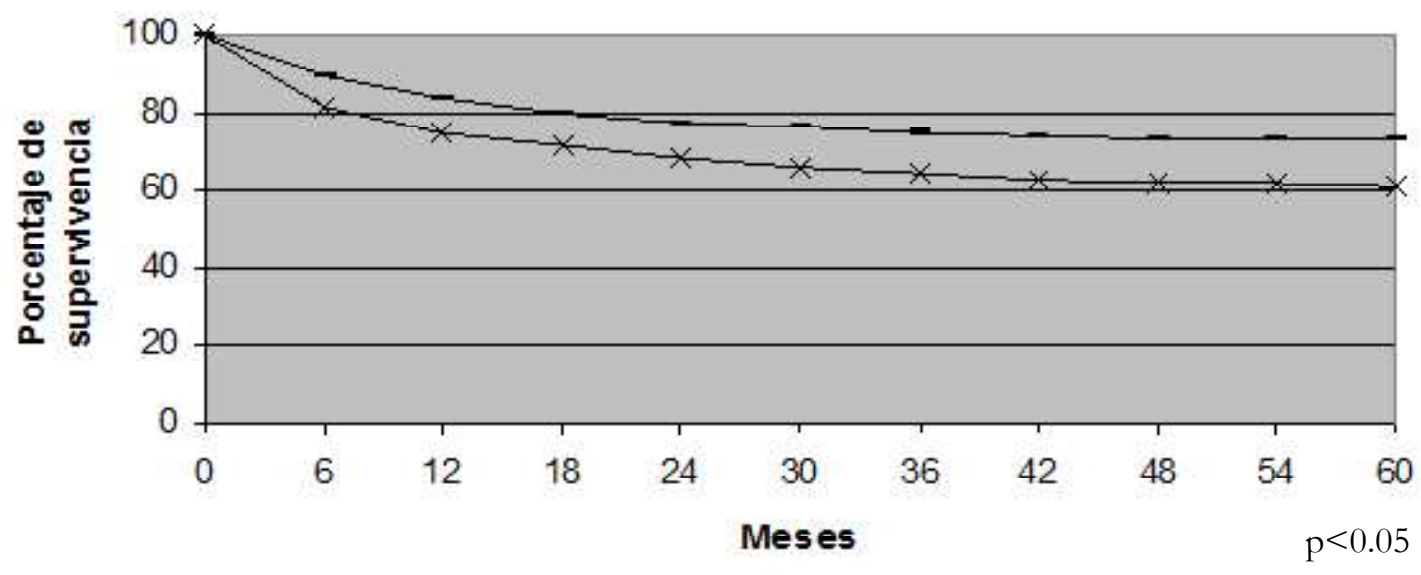

MUJERES $\longrightarrow$ HOMBRES

Gráfica 1. Curva de supervivencia por el método de Kaplan Meier, por sexo. ION SA, Manizales. 1995-2004

mixtas de toxicidad al mismo entre las que sobresalen las hematológicas y las localizadas en las mucosas. La supervivencia global de los pacientes se encuentra entre $61.2 \%$ y $72.3 \%$ a 5 años de observación.

Conflicto de intereses. Los autores declaran que no hay conflicto de intereses en este trabajo descriptivo ni se comprometen los intereses de la institución sede de la investigación, así como tampoco se mencionan marcas comerciales de ningún tipo.

\section{Agradecimientos}

A todos los empleados y funcionarios del Instituto Oncológico ION SA de Manizales, Colombia, por su colaboración en el suministro de la información que condujo a este trabajo.

\section{Referencias}

1. Ferlay J, Shin HR, Bray F, Forman D, Mathers C, Parkin DM. Estimates of worldwide burden of cancer in 2008: GLOBOCAN 2008. Int J Cancer. 2010; 127: 2893-917.

2. SEER. Cancer statistics review. Bethesda: National Cancer Institute. [Acceso: 2010 June 01] Disponible en: http:// seer.cancer.gov/csr/1975_2007/
3. Universidad de Caldas. Registro Poblacional de Cáncer de Manizales. Caldas año 2007. Quinquenio 2003-2007. Manizales: RPC.

4. CarolT, Lena-Marie P, Lesley F. D, Mirjam AGS. Symptom prevalence, intensity, and sistress in patients with inoperable lung cancer in relation to time of death. JCO. 2007; 25 (34): 5381-9.

5. De Vita V. Cancer principles and practice of oncology. 7th ed. Philadelphia: Lippincott Williams-Wilkins; 2005.

6. Anderson WF, Jatoi I, Tse J, Rosenberg PS. Male breast cancer: a population-based comparison with female breast cancer. J Clin Oncol. 2010; 28: 232-9.

7. Mohar A, Frías-Mendivil M, Suchil-Bernal L, Mora-Macías T, de la Garza JG. Epidemiologia descriptiva de cáncer en el Instituto Nacional de Cancerología de México. Salud Publica Mex. 1997; 39: 253-8.

8. Bravo-Cañón MA. Cáncer de mama en el hombre. XXIV Congreso Nacional, I Congreso Internacional de Oncología, Acapulco 2005. Memorias abs. p. 70.

9. Albano JD, Ward E, Ahmedin J, Anderson R, Cokkinides VE, Murray T, et al. Cancer mortality in the United States by education level and race. JNCI. 2007; 11: 1384-4.

10. Peeters KCMJ, van de Velde CJH, Leer JWH, Martijn H, Junggeburt JMC, Klein Kranenbarg E, et al. Late side effects of short-course preoperative radiotherapy combined with total mesorectal excision for rectal cancer: Increased bowel dysfunction in irradiated patients. A Dutch Colorectal Cancer Group Study. JCO. 2005; 23 (25): 6199-6. 